\title{
Thunbergia alata inhibits inflammatory responses through the inactivation of ERK and STAT3 in macrophages
}

\author{
YOUNG-CHANG CHO ${ }^{1}$, YE RANG KIM ${ }^{1}$, BA REUM KIM ${ }^{1}$, TRAN THE BACH $^{2}$ and SAYEON CHO ${ }^{1}$ \\ ${ }^{1}$ Laboratory of Molecular and Pharmacological Cell Biology, College of Pharmacy, Chung-Ang University, \\ Seoul 06974, Republic of Korea; ${ }^{2}$ Vietnam Academy of Science and Technology (VAST), Hanoi 10307, Vietnam
}

Received August 10, 2015; Accepted September 5, 2016

DOI: $10.3892 /$ ijmm.2016.2746

\begin{abstract}
Thunbergia alata (Acanthaceae) has been used traditionally to treat various inflammatory diseases such as fever, cough and diarrhea in East African countries including Uganda and Kenya. However, systemic studies elucidating the anti-inflammatory effects and precise mechanisms of action of $T$. alata have not been conducted, to the best of our knowledge. To address these concerns, we explored the anti-inflammatory effects of a methanol extract of T. alata (MTA) in macrophages. Non-cytotoxic concentrations of MTA $(\leq 300 \mu \mathrm{g} / \mathrm{ml})$ inhibited nitric oxide (NO) production in lipopolysaccharide (LPS)-stimulated RAW 264.7 macrophages by transcriptional regulation of inducible NO synthase in a dose-dependent manner. The expression of cyclooxygenase-2, the enzyme responsible for the production of prostaglandin $E_{2}$, was unchanged by MTA at the mRNA and protein levels. MTA treatment inhibited interleukin (IL)-6 production and decreased the mRNA expression of pro-inflammatory cytokines, including $I L-6$ and $I L-1 \beta$. Tumor necrosis factor- $\alpha$ production and mRNA expression were not regulated by MTA treatment. The decreased production of inflammatory mediators by MTA was followed by the reduced phosphorylation of extracellular signal-regulated kinase (ERK) and signal transducer and activator of transcription 3 (STAT3). MTA treatment had no effect on activity of other mitogen-activated protein kinases (MAPKs), p38, c-Jun $\mathrm{N}$-terminal kinase (JNK), and nuclear factor- $\kappa \mathrm{B}(\mathrm{NF}-\kappa \mathrm{B})$. These results indicate that MTA selectively inhibits the excessive production of inflammatory mediators in LPS-stimulated murine macrophages by reducing the activity of ERK and STAT3, suggesting that MTA plays an important inhibitory role in the modulation of severe inflammation.
\end{abstract}

Correspondence to: Professor Sayeon Cho, Laboratory of Molecular and Pharmacological Cell Biology, College of Pharmacy, Chung-Ang University, 84 Heukseok-Ro, Dongjak-Gu, Seoul 06974 , Republic of Korea

E-mail: sycho@cau.ac.kr

Key words: Thunbergia alata, nitric oxide, interleukin 6, tumor necrosis factor- $\alpha$, extracellular signal-regulated kinase, signal transducer and activator of transcription 3

\section{Introduction}

Inflammation is a defense mechanism of the innate immune system against invading agents such as bacteria, viruses, and fungi (1-4). Defense processes in the innate immune system are mediated by the production of various inflammatory mediators, such as nitric oxide (NO), and prostaglandin $\mathrm{E}_{2}\left(\mathrm{PGE}_{2}\right)$, as well as the expression of various pro-inflammatory cytokines, such as interleukin (IL)-1 $\beta$, IL-6, and tumor necrosis factor (TNF)- $\alpha$ (5-10). However, excessive amounts of these mediators cause severe inflammatory diseases, including septic shock, rheumatoid arthritis, systemic lupus erythematosus, cancer and inflammatory bowel disease, although the enhanced production of inflammatory mediators is important for host defense against external stimuli (10-16). Macrophages are the major cells that induce inflammatory responses by producing the various inflammatory mediators listed above. Therefore, investigation of the agents that inhibit the excessive production of inflammatory mediators in activated macrophages may be a viable strategy for developing effective anti-inflammatory agents.

Thunbergia alata (Acanthaceae), commonly known as Black-eyed Susan vine, is a native plant of East Africa and has been naturalized in other parts of the world, including Brazil, Hawaii, Eastern Australia, and Southern USA. This plant was traditionally used to treat inflammatory diseases. In Uganda, mild infusions of the leaves and stems of T. alata are orally administered to treat fever and malaria (17). Moreover, the infusion of leaves was also introduced to treat diarrhea (18). Jeruto et al reported that cough, flu, and backache were relieved by the oral administration of the infusion of T. alata leaves in Kenya (19). Other researchers reported that in Kenya, topical application of pounded leaves of T. alata was successful in the treatment of boils on the skin (20). Furthermore, several studies have elucidated the pharmacological properties of T. alata including antimicrobial, antiviral, antifungal and sun protective effects (21-23). According to phytochemical research, T. alata contains phenolic compounds, such as caffeoylmalic, feruloylmalic, and $p$-coumaroylmalic acids, iridoid glucosides such as alatoside and thunaloside, stilbericoside, 6-epi-stilbericoside and thunbergioside $(24,25)$. Of these compounds, caffeoylmalic acid has been reported to exert anti-inflammatory, antioxidant and anti-spasmodic effects (26-28). 
Although this plant has been used traditionally and evaluated for its pharmacological activities, no systemic studies on the immunomodulatory effects of the extract are available. In the present study, we aimed to confirm the ethnopharmacological benefits of T. alata in severe inflammatory states using lipopolysaccharide (LPS)-activated macrophages. Furthermore, we aimed to elucidate the precise mechanism of action responsible for the anti-inflammatory effects of $T$. alata as there are no prior reports regarding the mechanism of action of the methanol extract of T. alata (MTA).

\section{Materials and methods}

Preparation of MTA. A methanol extract (voucher no: KRIB0043981) of T. alata (Acanthaceae) collected from Lam Dong(Vietnam) was purchased from the International Biological Material Research Center [http://www.ibmrc.re.kr, Korea Research Institute of Bioscience and Biotechnology (KRIBB), Daejeon, Korea]. The concentrated methanol extract was manufactured according to a standard protocol of KRIBB. Briefly, the leaves and stems of plants ( $>1 \mathrm{~kg}$ by dry weight) were dried at room temperature (RT), treated with methanol (HPLC grade), and sonicated several times at $50^{\circ} \mathrm{C}$ for 3 days. The extracts were filtered to remove solid substances and concentrated with reduced pressure at $50^{\circ} \mathrm{C}$. A stock solution $(200 \mathrm{mg} / \mathrm{ml})$ of the extract was prepared in dimethylsulfoxide (DMSO), and this was stored at $-20^{\circ} \mathrm{C}$ prior to use.

Cell culture. RAW 264.7 macrophages, a mouse monocyte/macrophage cell line, were obtained from the American Type Cell Collection (ATCC; Manassas, VA, USA) and cultured in Dulbecco's modified Eagle's medium (DMEM) containing $10 \%$ fetal bovine serum (FBS) (both from GE Healthcare, Milwaukee, WI, USA), $50 \mathrm{U} / \mathrm{ml}$ penicillin, and $50 \mu \mathrm{g} / \mathrm{ml}$ streptomycin (Gibco-BRL, Grand Island, NY, USA) at $37^{\circ} \mathrm{C}$ in humidified air containing $5 \% \mathrm{CO}_{2}$.

Antibodies. Rabbit anti-inhibitor of $\kappa \mathrm{B}(\mathrm{I} \kappa \mathrm{B}) \alpha(\mathrm{sc}-371)$, anti-p38 (sc-535), anti-c-Jun N-terminal kinase (JNK; sc-571), anti-signal transducer and activator of transcription 3 (STAT3; sc-482), and anti-glyceraldehyde 3-phosphate dehydrogenase (GAPDH; sc-25778) antibodies were purchased from Santa Cruz Biotechnology (Santa Cruz, CA, USA). Rabbit anti-inducible NO synthase (iNOS; 2982), anti-cyclooxygenase (COX)-2 (4842), anti-phospho IкB $\alpha$ (2859), anti-phospho p38 (9212), anti-extracellular signal-regulated kinase (ERK; 9102), anti-phospho JNK (Thr184/Tyr185; 9251), and anti-phospho STAT3 (Tyr705; 9131) and mouse anti-phospho ERK (Thr202/Tyr204; 9106), were purchased from Cell Signaling Technology, Inc. (Danvers, MA, USA).

Cell viability assay. The RAW 264.7 macrophages were seeded in 96 -well plates $\left(4 \times 10^{4} /\right.$ well). Following adhesion overnight, cells were treated with LPS $(1 \mu \mathrm{g} / \mathrm{ml})$ and various concentrations of MTA for $24 \mathrm{~h}$. EZ-Cytox solution (Daeil Lab, Seoul, Korea), containing a water soluble tetrazolium salt, was added to each well for $2 \mathrm{~h}$ at $37^{\circ} \mathrm{C}$ and $100 \mu \mathrm{l}$ of supernatants were transferred to a new 96-well plate. The absorbance was measured at $450 \mathrm{~nm}$ (650 nm as a reference absorbance) using a Synergy H1 microplate reader (Bio-Tek Instruments, Winooski, VT, USA).
Nitrite assay. The RAW 264.7 macrophages seeded in 96-well plates $\left(4 \times 10^{4} /\right.$ well) overnight were incubated with MTA and LPS $(1 \mu \mathrm{g} / \mathrm{ml})$ for an additional $24 \mathrm{~h}$. Following incubation, the levels of NO were determined by assaying the culture supernatants for nitrite using Griess reagent (1\% sulfanilamide, $0.1 \% \mathrm{~N}$-1-naphthylenediamine dihydrochloride and $2.5 \%$ phosphoric acid). A standard curve for the calculation of $\mathrm{NO}$ production was acquired by measuring the absorbance of fixed $\mathrm{NaNO}_{2}$ standard solution. The absorbance was measured at $540 \mathrm{~nm}$ using a Synergy $\mathrm{H} 1$ microplate reader after incubation for $10 \mathrm{~min}$.

Enzyme-linked immunosorbent assay (ELISA). The RAW 264.7 cells were stimulated with LPS $(1 \mu \mathrm{g} / \mathrm{ml})$ and MTA for $24 \mathrm{~h}$. After stimulation, the supernatants were obtained, and a sandwich ELISA was performed to determine the quantities of TNF- $\alpha$ and IL-6 in culture supernatants using Ready-SET-Go! ${ }^{\circledR}$ ELISA kits (eBioscience, San Diego, CA, USA) with an antibody specific to each mediator. Briefly, the plate was pre-coated with coating antibody in the supplied buffer. Following incubation overnight at $4^{\circ} \mathrm{C}$, the plate was washed with $1 \mathrm{X}$ phosphate-buffered saline Tween (PBST) several times and treated with $1 \mathrm{X}$ assay diluents for $1 \mathrm{~h}$ to block non-specific binding. After removal of the assay diluents, each well was incubated with diluted supernatants or standard solutions for $2 \mathrm{~h}$ at RT. After washing with 1X PBST, the plate was treated with biotinylated secondary antibody solution for $1 \mathrm{~h}$. Subsequent replacement with horseradish peroxidase (HRP)-streptavidin solution was performed after washing several times. Following incubation at RT for $30 \mathrm{~min}$, the 3,3',5,5'-tetramethylbenzidine (TMB) substrate solution was added to a washed plate. The reaction was stopped by adding $1 \mathrm{~N}$ phosphoric acid $\left(\mathrm{H}_{3} \mathrm{PO}_{4}\right)$ after a 10 min incubation in dark conditions and the optical density of the individual wells was determined at $450 \mathrm{~nm}$ using a Synergy $\mathrm{H} 1$ microplate reader.

Semi-quantitative reverse transcription-polymerase chain reaction $(R T-P C R)$. RAW 264.7 macrophages were treated with LPS $(50 \mathrm{ng} / \mathrm{ml})$ and various concentrations of MTA. After $3 \mathrm{~h}$ incubation, total RNA was prepared from the cells using AccuZol (Bioneer, Daejeon, Korea) and reverse transcribed into complementary DNA (cDNA) using a TOPscript ${ }^{\mathrm{TM}}$ cDNA Synthesis kit (Enzynomics, Daejeon, Korea). PCR amplification of the cDNA was then performed using a PCR Premix (Bioneer). The sequences of PCR primers used were listed in our previous study (29). The PCR was run for 17-25 cycles of $94^{\circ} \mathrm{C}(30 \mathrm{sec}), 60^{\circ} \mathrm{C}(30 \mathrm{sec})$, and $72^{\circ} \mathrm{C}(30 \mathrm{sec})$ using a Bioer thermal cycler (Bioer Technology Co., Hangzhou, China). After amplification, $10 \mu \mathrm{l}$ of the PCR products was separated in $1.5 \%(\mathrm{w} / \mathrm{v})$ agarose gels and stained with ethidium bromide.

Reverse transcription-quantitative PCR (RT-qPCR). PCR amplification of the cDNA was performed using a qPCR Premix, iTaq ${ }^{\mathrm{TM}}$ Universal SYBR-Green Supermix (Bio-Rad, Hercules, CA, USA). The PCR was run for 40 cycles of denaturation at $94^{\circ} \mathrm{C}(5 \mathrm{sec})$ and annealing/extension at $60^{\circ} \mathrm{C}$ (30 sec) using a CFX Connect ${ }^{\mathrm{TM}}$ Real-Time thermal cycler (Bio-Rad). The results were normalized with multiple reference genes, $\beta$-actin and $G A P D H$, and were expressed as the relative gene expressions to the LPS-treated group (100\%). The PCR primers used were listed in our previous study (29). 
Preparation of total cell lysates. RAW 264.7 cells were treated with MTA and LPS $(1 \mu \mathrm{g} / \mathrm{ml})$ for $15 \mathrm{~min}$ [for IкBs and mitogen-activated protein kinases (MAPKs)] or $24 \mathrm{~h}$ (for iNOS, COX-2 and STAT3) and washed with ice-cold PBS several times. Cells were lysed in lysis buffer containing $0.5 \%$ IGEPAL $^{\circledR}$ CA- $630,0.5 \%$ Triton X-100, $150 \mathrm{mM} \mathrm{NaCl}$, $20 \mathrm{mM}$ Tris- $\mathrm{HCl}$ ( $\mathrm{pH} 8.0), 1 \mathrm{mM}$ ethylenediaminetetraacetic acid (EDTA), $1 \%$ glycerol, $1 \mathrm{mM}$ phenylmethylsulfonyl fluoride (PMSF), $10 \mathrm{mM} \mathrm{NaF}$, and $1 \mathrm{mM} \mathrm{Na}_{3} \mathrm{VO}_{4}$. The supernatants were collected in each microtube and centrifuged at $15,814 \mathrm{x}$ g for $30 \mathrm{~min}$ at $4^{\circ} \mathrm{C}$.

Western blot analysis. After boiling the mixture of lysates and sample buffers, aliquots of the samples were separated in a $10 \%$ sodium dodecyl sulfate (SDS)-polyacrylamide gel and transferred onto nitrocellulose membranes with transfer buffer [192 mM glycine, $25 \mathrm{mM}$ Tris- $\mathrm{HCl}(\mathrm{pH} \mathrm{8.8)}$, and $20 \% \mathrm{MeOH}(\mathrm{v} / \mathrm{v})]$. After blocking with 5\% non-fat dried milk in $1 \mathrm{X}$ Tris-buffered saline Tween (TBST) solution, the membrane was incubated overnight at $4{ }^{\circ} \mathrm{C}$ with the primary antibodies which were diluted in 5\% BSA- or $5 \%$ skim milk-1X TBST solution. After washing with TBST, each membrane was incubated for $1 \mathrm{~h}$ with secondary peroxidaseconjugated immunoglobulin G (IgG, 1:5,000). After washing several times, the protein bands were detected using an enhanced chemiluminescence (ECL) solution. In the present study, protein levels were quantified by scanning and analyzing western blots with LabWorks software (UVP Inc., Upland, CA, USA).

The blots shown in Fig. 2D, 3C and 5C were obtained from the same cell lysates and therefore the identical GAPDH blot was used. In addition, the blots shown in Fig. 5A and B were also obtained from the same cell lysates.

Statistical analysis and experimental replicates. The graph data are represented as the means \pm standard error of the mean (SEM). Determination of significant differences between experimental conditions were assessed by the Mann-Whitney U test which was performed using Prism 3.0 (GraphPad Software, San Diego, CA, USA) and $p<0.01$ was considered to indicate a statistically significant difference. The data from 9 replicates were analyzed, including three independent experiments with three replicates in each.

\section{Results}

Determination of the non-cytotoxic concentration of MTA in macrophages. We first examined the effect of MTA on the cell viability of RAW 264.7 macrophages in order to determine the maximal effective concentration that exhibits no cytotoxicity. RAW 264.7 cells were treated with various concentrations of MTA in the presence of LPS for $24 \mathrm{~h}$ and cell viability was accessed by measuring the formation of formazan. As shown in Fig. 1, cell viability did not decrease up to $300 \mu \mathrm{g} / \mathrm{ml}$ of MTA. However, a notable reduction in cell viability was observed at a concentration of $400 \mu \mathrm{g} / \mathrm{ml}$ of MTA. We subsequently performed all experiments using $300 \mu \mathrm{g} / \mathrm{ml}$ of MTA to exclude the possibility that the anti-inflammatory effect of MTA in activated macrophages was induced through a cytotoxic effect.

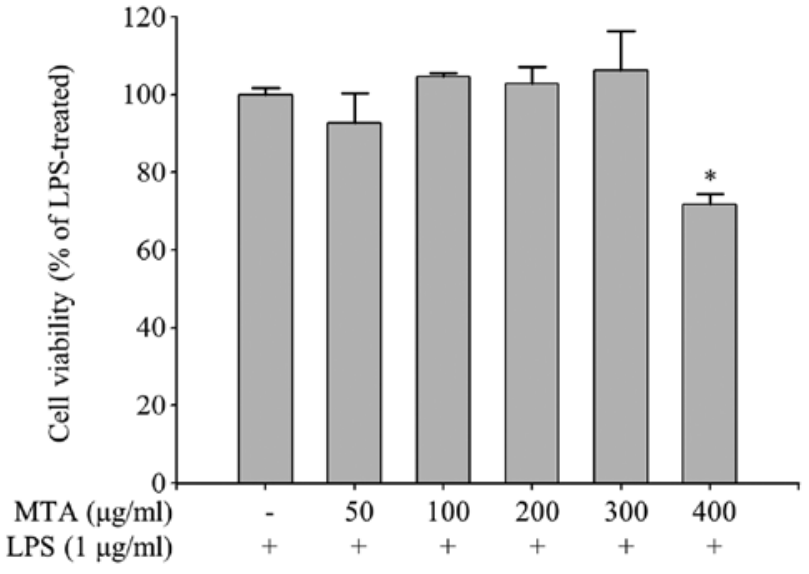

Figure 1. Effects of a methanol extract of Thunbergia alata (MTA) on cell viability. RAW 264.7 macrophages were treated simultaneously with lipopolysaccharide (LPS, $1 \mu \mathrm{g} / \mathrm{ml})$ and MTA $(50,100,200,300$, and $400 \mu \mathrm{g} / \mathrm{ml})$. Following incubation for $24 \mathrm{~h}$, cell viability was measured using the EZ-Cytox reagent. Cell viability was compared to that of the LPS-treated group. Data represent the means \pm SEM. ${ }^{*} \mathrm{p}<0.01$ relative to the LPS-treated control.

Inhibitory effect of MTA on the production of NO in $L P S$-stimulated macrophages. To evaluate the anti-inflammatory effects of MTA in macrophages, NO production and the expression of iNOS, the enzyme responsible for NO production, were measured in LPS-stimulated RAW 264.7 macrophages. LPS stimulation highly induced the production of $\mathrm{NO}$, as shown in Fig. 2A. The induction of $\mathrm{NO}$ was gradually reduced by MTA in a dose-dependent manner; and the quantity of NO produced at a concentration of $300 \mu \mathrm{g} / \mathrm{ml}$ of MTA was as low as that of the unstimulated control. We then examined whether MTA regulates the expression of iNOS at the mRNA and protein level since iNOS is a key enzyme for the production of NO in LPS-stimulated macrophages. RT-qPCR analysis revealed that LPS-induced expression of $i N O S$ was alleviated by MTA treatment in a dose-dependent manner (Fig. 2B); and semi-quantitative PCR result produced a similar profile to the qPCR data (Fig. 2C). Furthermore, the increased protein expression of iNOS through LPS treatment was dose-dependently reduced by MTA treatment (Fig. 2D). These results suggest that MTA negatively regulates the production of NO by regulating iNOS at the transcriptional level.

No effect of MTA on the expression of COX-2 in $L P S$-stimulated macrophages. Since COX-2 is the enzyme responsible for the production of $\mathrm{PGE}_{2}$, which induces fever during inflammation, we explored the anti-inflammatory effect of MTA by evaluating the mRNA and protein expression of COX-2. As shown in Fig. 3A and B, LPS induced $C O X-2$ gene expression but MTA treatment did not lead to suppression of LPS-induced $C O X-2$ expression. Similar to the RT-qPCR and semi-quantitative PCR data, MTA did not regulate LPS-induced COX-2 expression at the protein level (Fig. 3C). The majority of natural extracts that exhibit anti-inflammatory effects have been shown to suppress $\mathrm{PGE}_{2}$ production through the inhibition of COX-2 expression $(30,31)$. These results suggest that MTA does not regulate the production of PGE2 since COX-2 expression in activated macrophages is not inhibited by MTA. 

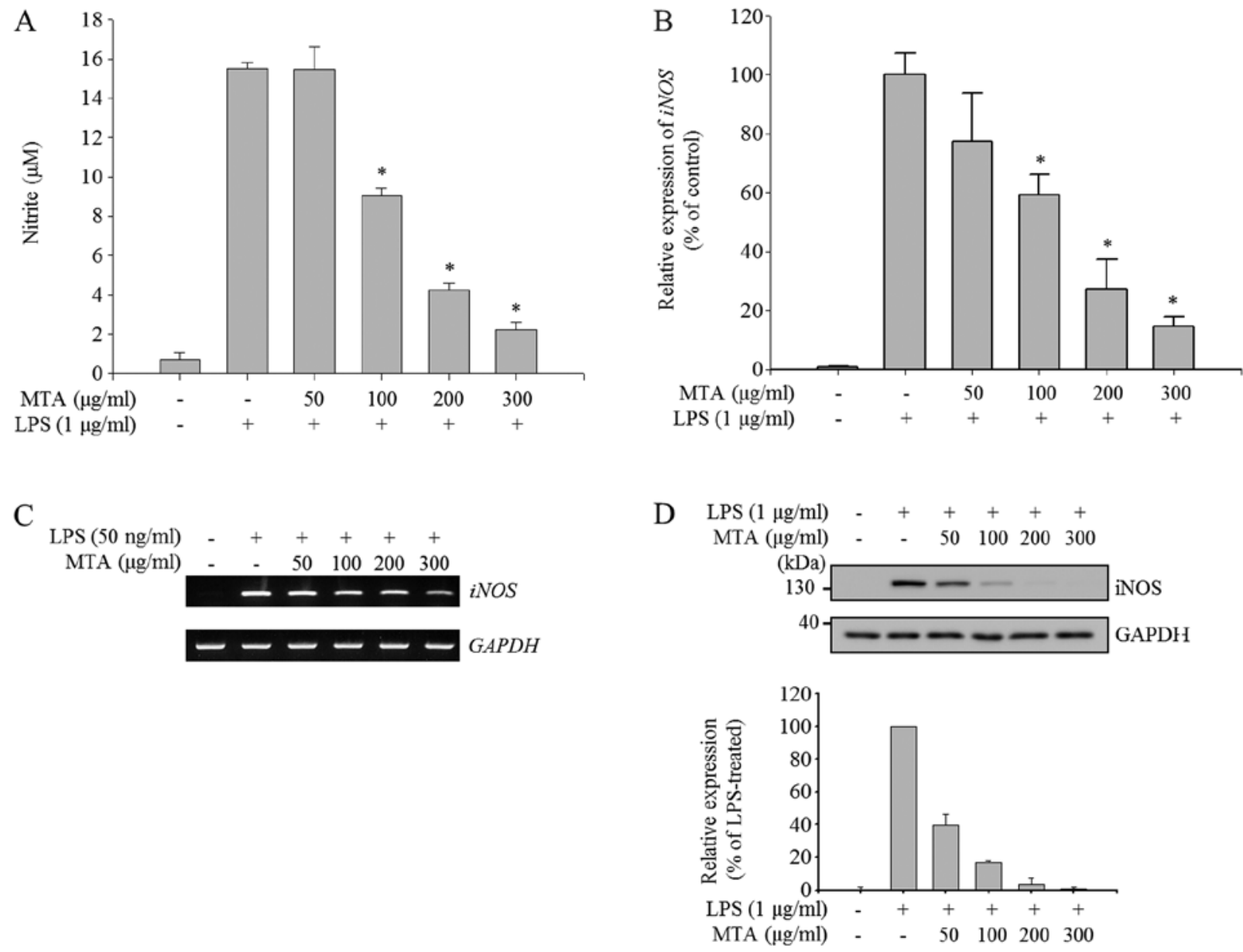

Figure 2. Inhibitory effects of a methanol extract of Thunbergia alata (MTA) on the production of nitric oxide (NO). RAW 264.7 macrophages were treated simultaneously with lipopolysaccharide (LPS) and MTA $(50,100,200$, and $300 \mu \mathrm{g} / \mathrm{ml})$ for the indicated times. (A) After $24 \mathrm{~h}$ of treatment, NO secretion in the supernatants was measured using the Griess reagent. Secreted quantities of NO were calculated using a standard curve for the nitrite standard solution. Data represent the means \pm SEM. ${ }^{*}$ p $<0.01$ relative to the LPS-treated control group. (B and C) After $6 \mathrm{~h}$ of treatment, total RNA was extracted and reverse transcribed to cDNA. (B) Inducible NO synthase ( $i N O S$ ) was amplified by reverse transcription-quantitative polymerase chain reaction (RT-qPCR), and the expression of $i N O S$ in each sample was compared with that in the LPS-treated group. Data represent the means \pm SEM. "p $<0.01$ relative to the LPS-treated control group. (C) iNOS was amplified by semi-quantitative PCR and detected using a gel documentation system. Glyceraldehyde-3-phosphate dehydrogenase (GAPDH) was used as an internal control. (D) Total cell lysates were prepared after $24 \mathrm{~h}$ of treatment and subjected to western blot analysis. iNOS protein expression was detected using an enhanced chemiluminescence reagent. Expression levels were quantified by analysis using LabWorks software and normalized to the corresponding GAPDH levels. Relative expression levels of iNOS are represented as a bar graph (lower panel).

Differential regulation of inflammatory cytokine production by MTA in LPS-stimulated macrophages. To further investigate the anti-inflammatory effects of MTA in activated macrophages, the profiles of proinflammatory cytokines, IL-1 $\beta$, IL- 6 , and TNF- $\alpha$, were measured in the presence or absence of MTA in LPS-stimulated RAW 264.7 macrophages. Cytokine secretion, including IL- 6 and TNF- $\alpha$, was highly increased by LPS treatment (Fig. 4A and B). Notably, MTA treatment specifically inhibited the production of IL-6 in a dose-dependent manner but did not regulate that of TNF- $\alpha$ (Fig. 4A and B). To determine whether the effect of MTA on the production of pro-inflammatory cytokines is closely regulated at the transcriptional level, the mRNA expression of pro-inflammatory cytokines was assessed in the presence or absence of MTA in LPS-treated RAW 264.7 macrophages. As shown in Fig. 4C, RT-qPCR analysis revealed that MTA treatment inhibited the LPS-stimulated expression of $I L-6$ and $I L-1 \beta$ but did not reduce that of TNF- $\alpha$. Similarly, semi-quantitative PCR analysis showed that MTA selectively reduced the mRNA expression of $I L-6$ and $I L-1 \beta$ (Fig. 4D). Taken together, these results suggest that MTA negatively regulates the LPS-induced production of pro-inflammatory cytokines by transcriptional repression of $I L-6$ and $I L-1 \beta$ but not $T N F-\alpha$.

Inhibitory effect of MTA on the activation of ERK and STAT3 in LPS-stimulated macrophages. The production of inflammatory mediators by LPS in macrophages is mainly induced through the subsequent activation of nuclear factor $-\mathrm{\kappa B}(\mathrm{NF}-\mathrm{\kappa B})$ and MAPKs, including p38, ERK, and JNK. The dissocia-

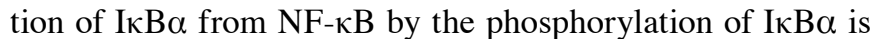
required for the activation of NF- $\mathrm{kB}$, and subsequent translocation of NF- $\kappa \mathrm{B}$ leads to the activation of inflammatory target genes. We therefore measured the phosphorylation levels of $\mathrm{I} \kappa \mathrm{B} \alpha$ in order to examine whether the inhibitory effects of MTA in LPS-stimulated macrophages occur through the $\mathrm{NF}-\kappa \mathrm{B}$ signaling pathway. As shown in Fig. 5A, LPS treatment induced the phosphorylation of I $\mathrm{B} \alpha$ approximately two-fold 

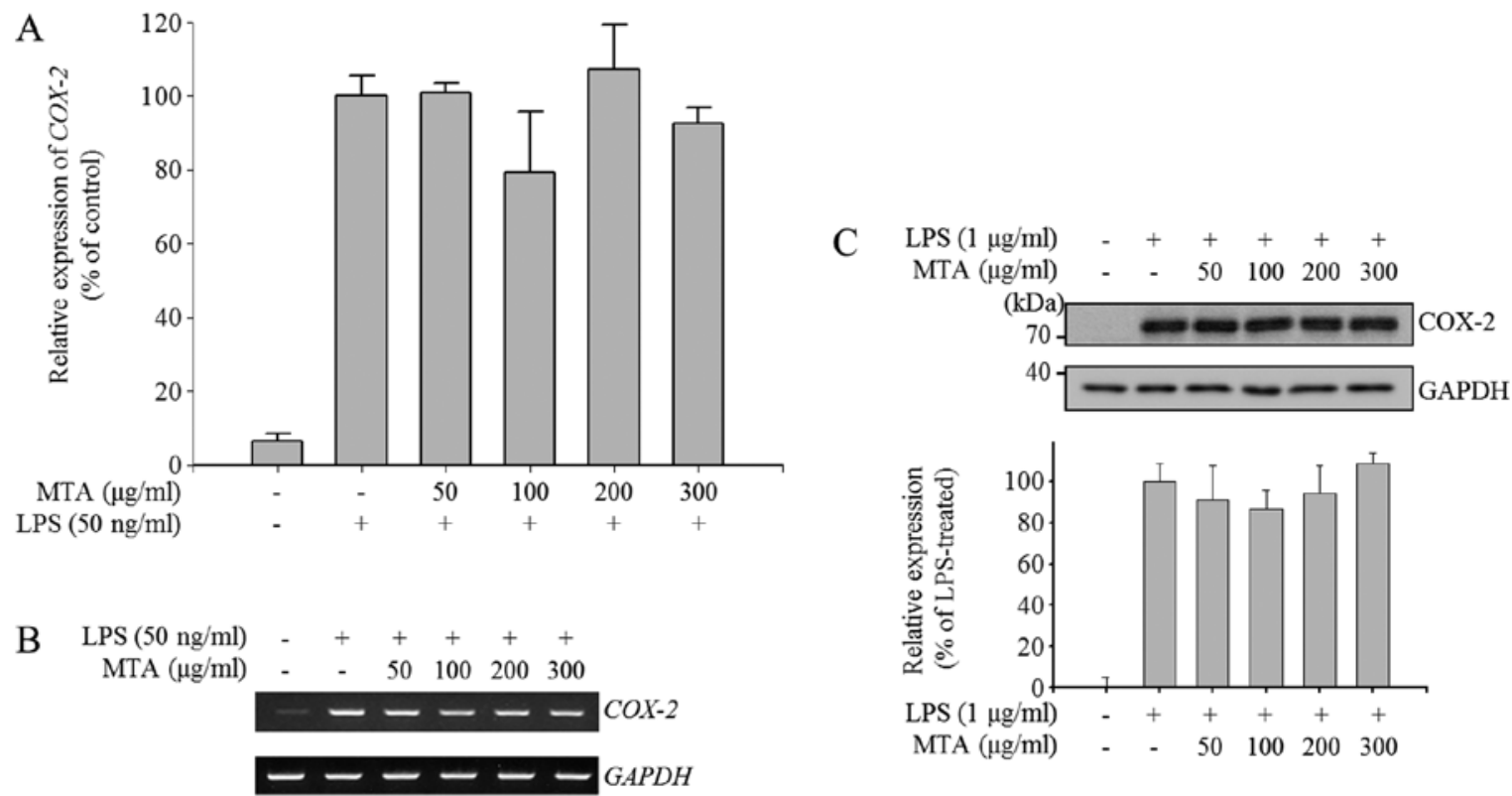

Figure 3. Effects of a methanol extract of Thunbergia alata (MTA) on the expression of cyclooxygenase-2 (COX-2). RAW 264.7 macrophages were treated simultaneously with lipopolysaccharide (LPS) and MTA $(50,100,200$, and $300 \mu \mathrm{g} / \mathrm{ml})$ for the indicated times. (A and B) After $6 \mathrm{~h}$ of treatment, total RNA was extracted and reverse transcribed to complementary DNA (cDNA). (A) COX-2 was amplified by RT-qPCR, and the expression of $C O X-2$ in each sample was compared with that of the LPS-treated control. Data represent the means \pm SEM. (B) COX-2 was amplified by semi-quantitative PCR and detected using a gel documentation system. Glyceraldehyde-3-phosphate dehydrogenase (GAPDH) was used as an internal control. (C) Total cell lysates were prepared after $24 \mathrm{~h}$ treatment and subjected to western blot analysis. COX-2 protein expression was detected using an enhanced chemiluminescence reagent, and expression levels were normalized to levels of the GAPDH. Relative expression levels of COX-2 are represented as a bar graph (lower panel)
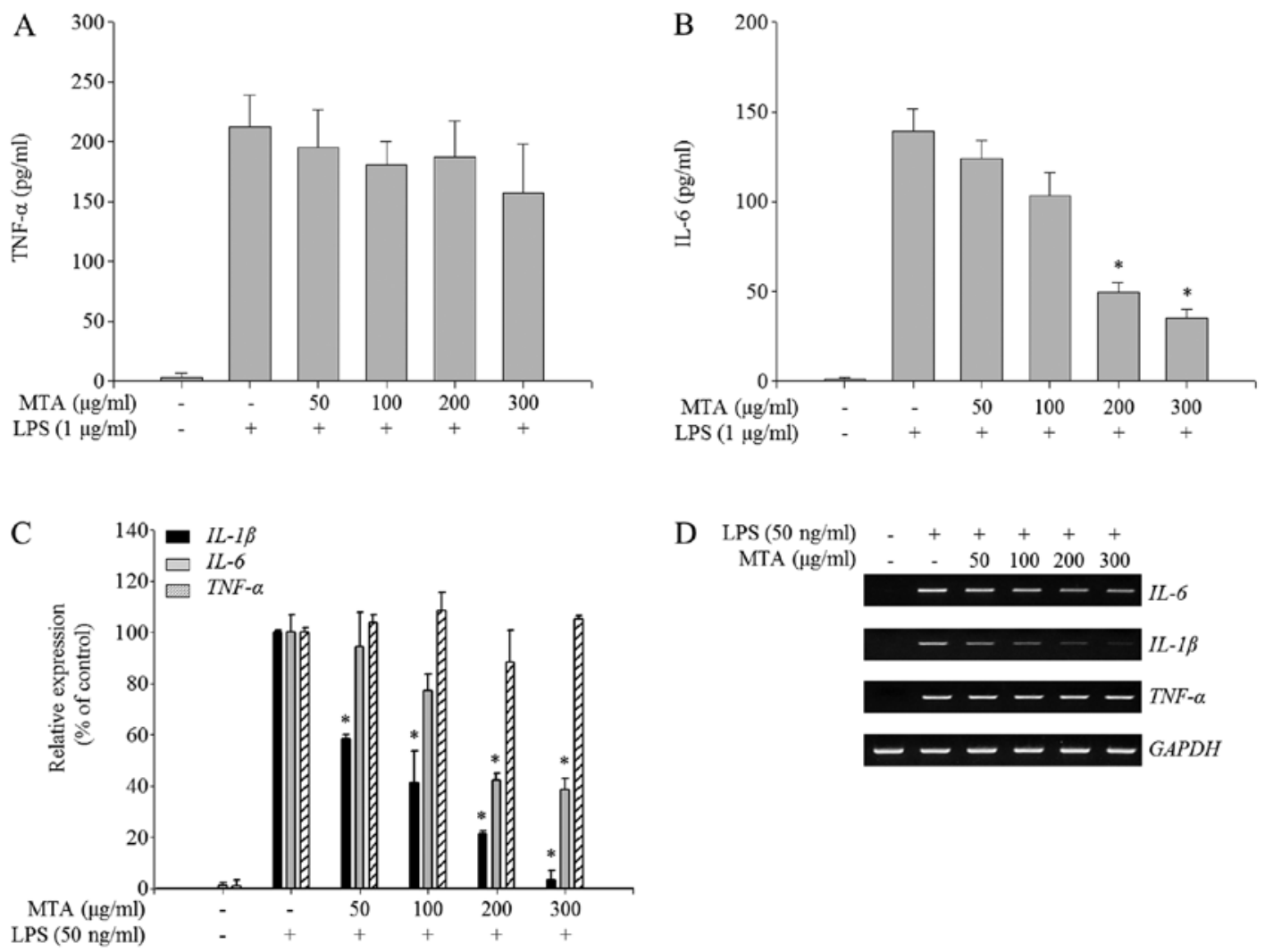

Figure 4. Inhibitory effect of a methanol extract of Thunbergia alata (MTA) on the production of pro-inflammatory cytokines. RAW 264.7 macrophages were treated simultaneously with lipopolysaccharide (LPS) and MTA $(50,100,200$, and $300 \mu \mathrm{g} / \mathrm{ml})$ for the indicated times. (A and B) After $24 \mathrm{~h}$ of treatment, enzyme-linked immunosorbent assays (ELISAs) were used to measure levels of (A) tumor necrosis factor (TNF)- $\alpha$ and (B) interleukin (IL)- 6 . The secretion of each cytokine was determined using a standard curve. Data represent the means \pm SEM. " $p<0.01$ relative to the LPS-treated control. (C and D) After $6 \mathrm{~h}$ of treatment, total RNA was extracted and reverse transcribed to cDNA. (C) $I L-1 \beta, T N F-\alpha$ and $I L-6$ were amplified by RT-qPCR, and the expression of $I L-1 \beta, T N F-\alpha$ and, $I L-6$ in each sample were compared to those of the LPS-treated control. Data represent the means \pm SEM. ${ }^{*} \mathrm{p}<0.01$ relative to the LPS-treated control. (D) $I L-1 \beta, T N F-\alpha$ and $I L-6$ were amplified by semi-quantitative PCR and detected using a gel documentation system. 
A

$\begin{array}{ccccccc}\operatorname{LPS}(1 \mu \mathrm{g} / \mathrm{ml}) & - & + & + & + & + & + \\ \operatorname{MTA}(\mu \mathrm{g} / \mathrm{ml}) & - & - & 50 & 100 & 200 & 300\end{array}$
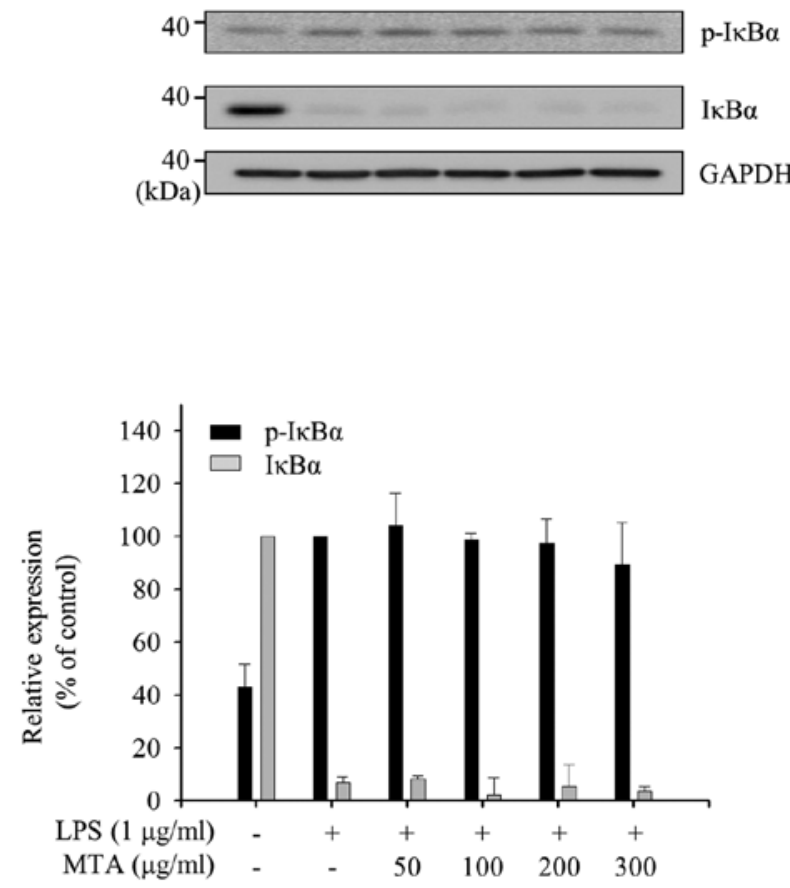

$\mathrm{C}$

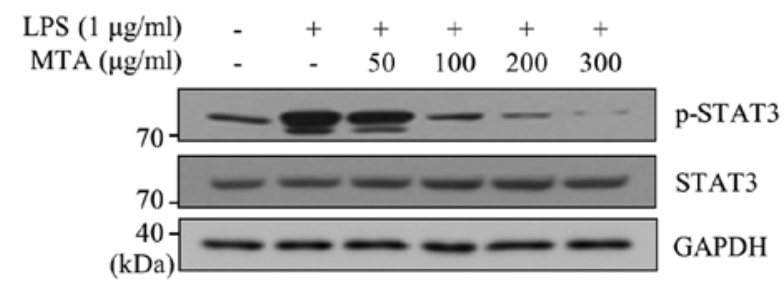

B
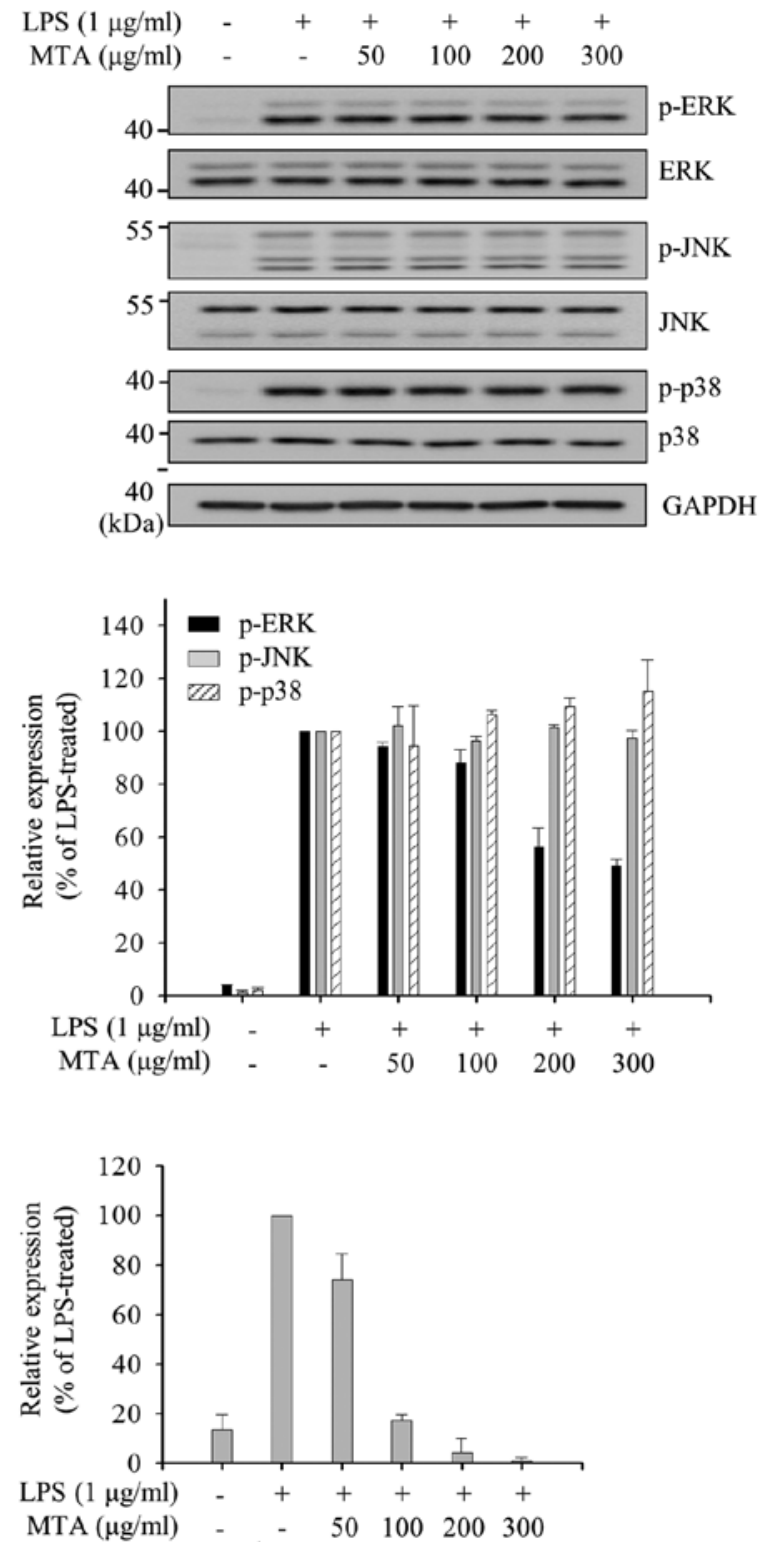

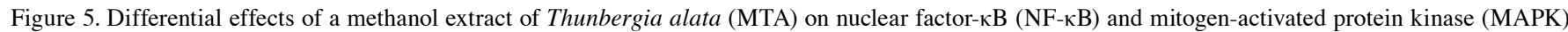
activation. RAW 264.7 macrophages were pre-treated with various concentrations of MTA (50, 100, 200, and $300 \mu \mathrm{g} / \mathrm{ml})$ for $1 \mathrm{~h}$ and then stimulated with lipopolysaccharide (LPS) for $15 \mathrm{~min}$ (A and B) or $24 \mathrm{~h}(\mathrm{C})$. Total cell lysates were prepared and subjected towestern blot analysis. The expression of (A) phosphorylated inhibitor of $\kappa \mathrm{B}$ protein $(\mathrm{p}-\mathrm{I} \kappa \mathrm{B} \alpha$ ), I $\mathrm{IB} \alpha,(\mathrm{B}) \mathrm{p}$-c-Jun N-terminal kinase (JNK), JNK, p-extracellular signal-regulated kinase (ERK), ERK, p-p38, and p38 were detected using specific antibodies. Relative expression levels of $\mathrm{I} \kappa \mathrm{B} \alpha$ and $\mathrm{p}$-I $\kappa \mathrm{B} \alpha$ were normalized to glyceraldehyde-3-phosphate dehydrogenase (GAPDH) levels. Levels of p-MAPKs were normalized to the corresponding MAPK levels. Quantitative analyses of phosphorylation and protein levels are shown after normalization (lower panels). (C) Total cell lysates were prepared after $24 \mathrm{~h}$ treatment and subjected to western blot analysis. The phosphorylation and total protein levels of signal transducer and activator of transcription 3 (STAT3) was detected using specific antibodies. Levels of p-STAT3 were normalized to the total STAT3 levels and were shown as a bar graph representing ratio to LPS-treated group (right panel).

compared to the untrated group and reduced the expression of I $\mathrm{B} \alpha$ due to phosphorylation-dependent degradation. However, MTA treatment did not change the levels of $\mathrm{p}-\mathrm{I} \kappa \mathrm{B} \alpha$ and $\mathrm{I} \kappa \mathrm{B} \alpha$, suggesting that MTA does not play a regulatory role in $\mathrm{NF}-\kappa \mathrm{B}$ signaling. The regulation of MAPK signaling, another major LPS-induced inflammatory signaling pathway, by MTA was also investigated. As shown in Fig. 5B, the LPS-induced phosphorylation of ERK was reduced by MTA without changing the protein level of ERK, but the phosphorylations of other MAPKs, JNK and p38, were unchanged by MTA. Collectively, these results suggested that MTA exhibits anti-inflammatory properties by inhibiting ERK signaling.
The differential regulation of pro-inflammatory cytokines by MTA may be due to different transcription factor binding regions in the gene promoter of each cytokine. Previous studies revealed that the STAT protein-binding region is contained in the IL- 6 and IL- $1 \beta$ promoter regions but not in the TNF- $\alpha$ promoter region (32). This suggests that specific regulation of IL- 6 and IL-1 $\beta$ production by MTA in macrophages may be regulated through the inactivation of STAT signaling. We accordingly measured the change in STAT3 phosphorylation status at Tyr705 following MTA treatment of LPS-stimulated RAW 264.7 macrophages since STAT3 signaling is activated through phosphorylation at Tyr705. LPS-induced 


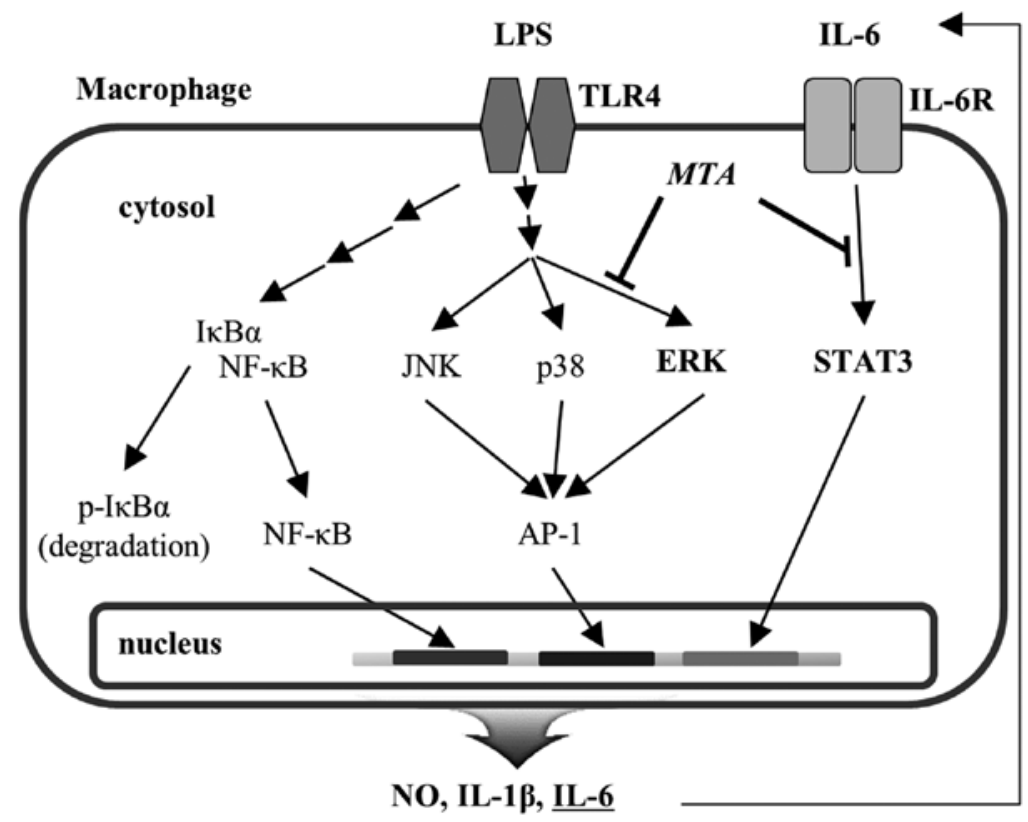

Figure 6. The putative pathway for the regulation of inflammatory mediators by a methanol extract of Thunbergia alata (MTA). MTA inhibits inflammatory responses by decreasing extracellular signal-regulated kinase (ERK) and signal transducer and activator of transcription 3 (STAT3) activity in lipopolysaccharide (LPS)-stimulated macrophages.

phosphorylation of STAT3 at Tyr705 was decreased by MTA in a dose-dependent manner (Fig. 5C). This result suggests that the differential regulation of pro-inflammatory cytokines by MTA is due to negative modulation of STAT3 activation.

\section{Discussion}

It is necessary to develop novel anti-inflammatory agents since steroidal drugs such as glucocorticoids have severe adverse effects (33-36). Non-steroidal anti-inflammatory drugs (NSAIDs) such as aspirin and indomethacin were developed and many studies have focused on the development of novel drugs capable of functioning as anti-inflammatory cytokine agents (37). Due to these concerns, native plants and their active constituents are receiving greater attention nowadays since anecdotal evidence regarding traditional usage as a medicine indicates the development of relatively fewer side effects (38). Many phytochemicals have been identified as anti-inflammatory drug candidates and are under investigation for clinical use $(39,40)$. In this study, we examined the anti-inflammatory effects of MTA and the underlying molecular mechanism responsible for these effects.

The activation of NF- $\kappa \mathrm{B}$ and MAPKs is primed by LPS ligation to Toll-like receptors. However, the activities of $\mathrm{NF}-\kappa \mathrm{B}$ and MAPKs are regulated by different upstream kinases, such as $\mathrm{I} \kappa \mathrm{B}$ kinases for NF- $\kappa \mathrm{B}$ and MAPK kinases for MAPKs. Therefore, the data in Fig. 5A and B suggest that MTA regulates ERK activation specifically for its anti-inflammatory properties without disturbing the activation of other MAPKs and $\mathrm{NF}-\kappa \mathrm{B}$ in LPS-stimulated macrophages. Previous studies that describe the differential regulation of MAPKs by anti-inflammatory compounds, support our hypothesis (41-43). Several components, such as curcumin, quercetin and resveratrol from plants, inhibit ERK activity to exhibit various pharmacological effects in inflammatory diseases (44-46).
TNF- $\alpha$ production increases through enhanced activator protein 1 (AP-1) AP-1 transcriptional activity by MAPKs. Therefore, as expected, LPS treatment induced TNF- $\alpha$ production in RAW 264.7 cells. However, LPS-induced TNF- $\alpha$ production was not inhibited by MTA treatment despite the reduced ERK activity (Figs. 4A and 5B). It remains unclear the reason why TNF- $\alpha$ induction was not suppressed through inhibition of ERK by MTA treatment. As shown in Fig. 5B, the phosphorylation of JNK and p38 was not inhibited by high concentrations of MTA $(300 \mu \mathrm{g} / \mathrm{ml})$. These data suggest that reduced AP-1 transcriptional activity through MTA-mediated ERK inhibition may be recovered due to the functional redundancy of JNK and p38 that are not inhibited by MTA.

As shown in the blots of Fig. 5B and C, the effects of MTA on the regulation of both ERK and STAT3 were notably different. MTA treatment at low doses almost completely inhibited the phosphorylation of STAT3 (Fig. 5C). However, phosphorylated ERK was only $50 \%$ reduced when LPS-stimulated cells were treated with the maximal concentration of MTA (Fig. 5B), suggesting that ERK is less sensitive to MTA than STAT3. Since the major regulatory pathway for STAT3 activation is mediated by autocrine IL- 6 via LPS-activated NF- $\kappa \mathrm{B}$ and MAPK signal transduction, these results suggest that MTA selectively inhibits the IL-6-mediated STAT3 pathway at low concentrations but inhibits both ERK and STAT3 pathways at high concentrations. The differential regulation of inflammatory mediators by MTA is likely due to the different actions that are regulated by different concentrations of MTA. The proposed regulatory mechanisms for the anti-inflammatory effects of MTA are shown in Fig. 6.

Collectively, the findings of the present study suggest that MTA selectively inhibits the production of various inflammatory mediators by reducing the activation of ERK and STAT3 signaling pathways in macrophages. Further studies are necessary in order to elucidate more fully the mechanisms of action of 
MTA involved in the regulation of severe inflammatory states. Although we elucidated the mechanism of action of MTA in murine macrophages as an inhibitor of ERK and STAT3 activation, it is also necessary to clarify the major phytochemicals that mediate the anti-inflammatory effects of MTA.

\section{Acknowledgements}

Th present study was supported by the National Research Foundation of Korea (NRF) grant funded by the Ministry of Science, ICT and Future Planning (NRF-2015R1A2A2A11001446, NRF-2015R1A5A1008958) and by the Ministry of Education, Science and Technology (NRF-2013R1A1A2062389).

\section{References}

1. Boscá L, Zeini M, Través PG and Hortelano S: Nitric oxide and cell viability in inflammatory cells: a role for NO in macrophage function and fate. Toxicology 208: 249-258, 2005.

2. Fujihara M, Muroi M, Tanamoto K, Suzuki T, Azuma H and Ikeda $\mathrm{H}$ : Molecular mechanisms of macrophage activation and deactivation by lipopolysaccharide: roles of the receptor complex. Pharmacol Ther 100: 171-194, 2003.

3. Fujiwara $\mathrm{N}$ and Kobayashi K: Macrophages in inflammation. Curr Drug Targets Inflamm Allergy 4: 281-286, 2005.

4. MacMicking J, Xie QW and Nathan C: Nitric oxide and macrophage function. Annu Rev Immunol 15: 323-350, 1997.

5. Jones BW, Heldwein KA, Means TK, Saukkonen JJ and Fenton MJ: Differential roles of Toll-like receptors in the elicitation of proinflammatory responses by macrophages. Ann Rheum Dis 60 (Suppl 3): iii6-iii12, 2001.

6. Jones BW, Means TK, Heldwein KA, Keen MA, Hill PJ, Belisle JT and Fenton MJ: Different Toll-like receptor agonists induce distinct macrophage responses. J Leukoc Biol 69: 1036-1044, 2001.

7. Rhee SH and Hwang D: Murine Toll-like receptor 4 confers lipopolysaccharide responsiveness as determined by activation of NF kappa B and expression of the inducible cyclooxygenase. J Biol Chem 275: 34035-34040, 2000.

8. Schroder K, Sweet MJ and Hume DA: Signal integration between IFNgamma and TLR signalling pathways in macrophages. Immunobiology 211: 511-524, 2006.

9. Stalińska K, Guzdek A, Rokicki M and Koj A: Transcription factors as targets of the anti-inflammatory treatment. A cell culture study with extracts from some Mediterranean diet plants. J Physiol Pharmacol 56 (Suppl 1): 157-169, 2005.

10. Szabó $\mathrm{C}$ and Thiemermann C: Regulation of the expression of the inducible isoform of nitric oxide synthase. Adv Pharmacol 34: 113-153, 1995.

11. Clancy RM, Amin AR and Abramson SB: The role of nitric oxide in inflammation and immunity. Arthritis Rheum 41: 1141-1151, 1998.

12. Guadagni F, Ferroni P, Palmirotta R, Portarena I, Formica V and Roselli M: Review. TNF/VEGF cross-talk in chronic inflammation-related cancer initiation and progression: an early target in anticancer therapeutic strategy. In Vivo 21: 147-161, 2007.

13. Kröncke KD, Fehsel K and Kolb-Bachofen V: Inducible nitric oxide synthase in human diseases. Clin Exp Immunol 113: 147-156, 1998.

14. Nathan C and Xie QW: Nitric oxide synthases: roles, tolls, and controls. Cell 78: 915-918, 1994.

15. Nathan C and Xie QW: Regulation of biosynthesis of nitric oxide. J Biol Chem 269: 13725-13728, 1994.

16. Nishimoto $\mathrm{N}$ and Kishimoto T: Interleukin 6: From bench to bedside. Nat Clin Pract Rheumatol 2: 619-626, 2006.

17. Hamill FA, Apio S, Mubiru NK, Mosango M, Bukenya-Ziraba R, Maganyi OW and Soejarto DD: Traditional herbal drugs of southern Uganda, I. J Ethnopharmacol 70: 281-300, 2000.

18. Tabuti JR, Lye KA and Dhillion SS: Traditional herbal drugs of Bulamogi, Uganda: plants, use and administration. J Ethnopharmacol 88: 19-44, 2003.

19. Jeruto P, Lukhoba C, Ouma G, Otieno D and Mutai C: An ethnobotanical study of medicinal plants used by the Nandi people in Kenya. J Ethnopharmacol 116: 370-376, 2008.
20. Okello SV, Nyunja RO, Netondo GW and Onyango JC: Ethnobotanical study of medicinal plants used by Sabaots of Mt. Elgon Kenya. Afr J Tradit Complement Altern Med 7: 1-10, 2009.

21. Patil SB, Lende MY, Thakur VS, Naikwade NS and Magdum CS: Sun protective activity of the hydroalchoholic extracts of two medicinal flowers. Asian J Pharm Res 2: 37-38, 2012.

22. Vlietinck AJ, Van Hoof L, Totté J, Lasure A, Vanden Berghe D, Rwangabo PC and Mvukiyumwami J: Screening of hundred Rwandese medicinal plants for antimicrobial and antiviral properties. J Ethnopharmacol 46: 31-47, 1995.

23. Jenifer S, Priya S, Laveena DK, Singh JS and Jeyasree J: Sensitivity patterns of some flowering plants against Salmonella typhi and Pseudomonas aeruginosa. J Pharm Pharm Sci 3: 1212-1220, 2014.

24. Housti F, Andary C, Gargadennec A and Amssa M: Effects of wounding and salicylic acid on hydroxycinnamoylmalic acids in Thunbergia alata. Plant Physiol Biochem 40: 761-769, 2002.

25. Damfort S, Frederiksen LB and Jensen SR: Alatoside and thunaloside, two iridoid glucosides from Thunbergia alata. Phytochemistry 35: 1259-1261, 1994.

26. Boegge SC, Kesper S, Verspohl EJ and Nahrstedt A: Reduction of ACh-induced contraction of rat isolated ileum by coptisine, (+)-caffeoylmalic acid, Chelidonium majus, and Corydalis lutea extracts. Planta Med 62: 173-174, 1996.

27. Kimura Y, Okuda H, Okuda T, Hatano T and Arichi S: Studies on the activities of tannins and related compounds, X. Effects of caffeetannins and related compounds on arachidonate metabolism in human polymorphonuclear leukocytes. J Nat Prod 50: 392-399, 1987.

28. Pearce G, Johnson S and Ryan CA: Purification and characterization from tobacco (Nicotiana tabacum) leaves of six small, wound-inducible, proteinase isoinhibitors of the potato inhibitor II family. Plant Physiol 102: 639-644, 1993.

29. Cho YC, Ju A, Kim BR and Cho S: Anti-inflammatory effects of Crataeva nurvala Buch. Ham. are mediated via inactivation of ERK but not NF-кB. J Ethnopharmacol 162: 140-147, 2015.

30. Lee CW, Park SM, Kim YS, Jegal KH, Lee JR, Cho IJ, Ku SK, Lee JY, Ahn YT, Son Y, et al: Biomolecular evidence of anti-inflammatory effects by Clematis mandshurica Ruprecht root extract in rodent cells. J Ethnopharmacol 155: 1141-1155, 2014.

31. Yu T, Lee S, Yang WS, Jang HJ, Lee YJ, Kim TW, Kim SY, Lee J and Cho JY: The ability of an ethanol extract of Cinnamomum cassia to inhibit Src and spleen tyrosine kinase activity contributes to its anti-inflammatory action. J Ethnopharmacol 139: 566-573, 2012.

32. Lee C, Lim HK, Sakong J, Lee YS, Kim JR and Baek SH: Janus kinase-signal transducer and activator of transcription mediates phosphatidic acid-induced interleukin (IL)-1beta and IL-6 production. Mol Pharmacol 69: 1041-1047, 2006.

33. Rubaltelli FF, Chiti G and Dani C: Adverse effects of prenatal glucocorticoid treatment in the preterm infant. Acta Biomed Ateneo Parmense 68 (Suppl 1): 35-38, 1997.

34. Münstedt K, Borces D, Bohlmann MK, Zygmunt $M$ and von Georgi R: Glucocorticoid administration in antiemetic therapy: is it safe? Cancer 101: 1696-1702, 2004.

35. Manelli F and Giustina A: Glucocorticoid-induced osteoporosis. Trends Endocrinol Metab 11: 79-85, 2000.

36. van Raalte DH, Ouwens DM and Diamant M: Novel insights into glucocorticoid-mediated diabetogenic effects: towards expansion of therapeutic options? Eur J Clin Invest 39: 81-93, 2009.

37. Dinarello CA: Anti-inflammatory agents: present and future. Cell 140: 935-950, 2010

38. Bassett IB, Pannowitz DL and Barnetson RS: A comparative study of tea-tree oil versus benzoylperoxide in the treatment of acne. Med J Aust 153: 455-458, 1990.

39. Naksuriya O, Okonogi S, Schiffelers RM and Hennink WE: Curcumin nanoformulations: a review of pharmaceutical properties and preclinical studies and clinical data related to cancer treatment. Biomaterials 35: 3365-3383, 2014.

40. Ranjan AP, Mukerjee A, Helson L, Gupta R and Vishwanatha JK: Efficacy of liposomal curcumin in a human pancreatic tumor xenograft model: inhibition of tumor growth and angiogenesis. Anticancer Res 33: 3603-3609, 2013.

41. Burk DR, Senechal-Willis P, Lopez LC, Hogue BG and Daskalova SM: Suppression of lipopolysaccharide-induced inflammatory responses in RAW 264.7 murine macrophages by aqueous extract of Clinopodium vulgare L. (Lamiaceae). J Ethnopharmacol 126: 397-405, 2009. 
42. Liu H, Bargouti M, Zughaier S, Zheng Z, Liu Y, Sangadala S, Boden SD and Titus L: Osteoinductive LIM mineralization protein-1 suppresses activation of NF-kappaB and selectively regulates MAPK pathways in pre-osteoclasts. Bone 46: $1328-1335,2010$.

43. Shan J, Fu J, Zhao Z, Kong X, Huang H, Luo L and Yin Z: Chlorogenic acid inhibits lipopolysaccharide-induced cyclooxygenase-2 expression in RAW264.7 cells through suppressing NF-kappaB and JNK/AP-1 activation. Int Immunopharmacol 9: 1042-1048, 2009.

44. Lin CW, Hou WC, Shen SC, Juan SH, Ko CH, Wang LM and Chen YC: Quercetin inhibition of tumor invasion via suppressing PKC delta/ERK/AP-1-dependent matrix metalloproteinase- 9 activation in breast carcinoma cells. Carcinogenesis 29: $1807-1815,2008$
45. Min Z, Kang L, Lin L, Jinghua F, Junna S and Baolin L: Resveratrol restores lysophosphatidylcholine-induced loss of endothelium-dependent relaxation in rat aorta tissue coinciding with inhibition of extracellular-signal-regulated protein kinase activation. Phytother Res 24: 1762-1768, 2010.

46. Wang J and Dong S: ICAM-1 and IL- 8 are expressed by DEHP and suppressed by curcumin through ERK and p38 MAPK in human umbilical vein endothelial cells. Inflammation 35 : 859-870, 2012. 